\title{
Diagnosis of Obstructive Sleep Apnea in Adults Using the Cardiopulmonary Coupling-Derived Software-Generated Apnea-Hypopnea Index
}

\author{
Min Young Seo (iD · Jun Yoo (iD · Sun Jin Hwang (D) $\cdot$ Seung Hoon Lee (iD \\ Division of Rhinology and Sleep Medicine, Department of Otorhinolaryngology-Head and Neck Surgery, Korea University Ansan Hospital, \\ Korea University College of Medicine, Ansan, Korea
}

Obstructive sleep apnea (OSA) is characterized by repetitive upper airway obstruction and apneic spells during sleep [1]. The standard diagnostic modality for OSA is polysomnography (PSG), and the presence and severity of OSA are determined using the apnea-hypopnea index (AHI) [2]. AHI values are derived from assessments of respiratory flow, oxygen desaturation, and arousal during sleep. Thus, a great amount of medical facilities, resources, and efforts are needed to perform PSG, which has spurred many physicians to attempt to develop simpler diagnostic modalities for OSA. With this goal in mind, we assessed sleep quality and stability using cardiopulmonary coupling (CPC) analysis [3,4]. Electrocardiogram (ECG)-based CPC analysis reflects sleep stability using cardiac regulation and respiratory variation [5]. In these studies, we found that the parameters related to sleep quality and stability generated from CPC analysis were significantly correlated with the AHI. Recently, Hilmisson et al. [6] published an article on sleep apnea diagnosis using a software-generated AHI (sAHI) derived from CPC analysis in children. They reported that the sAHI was comparable to the manual scoring of AHI from in-laboratory PSG studies and was effective for assessing OSA severity in children. Thus, in this study, we investigated the diagnostic value of the sAHI in adults using a cloud-based software technology, SleepImage (MyCardio, Denver, CO, USA).

We reviewed the medical records of 194 adult patients (age,

\footnotetext{
- Received September 10, 2020

Revised October 6, 2020

Accepted October 13, 2020

- Corresponding author: Seung Hoon Lee

Department of Otorhinolaryngology-Head and Neck Surgery, Korea

University Ansan Hospital, 123 Jeokgeum-ro, Danwon-gu, Ansan 15355, Korea

Tel: +82-31-412-5270, Fax: +82-31-412-5174

E-mail: shleeent@korea.ac.kr
}

18-72 years) with sleep-disordered breathing who underwent full-night in-laboratory PSG and CPC analysis concurrently between 2007 and 2013. We excluded patients who had insufficient sleep (total sleep time $<6$ hours), neuroactive drug usage, or concurrent significant medical comorbidities. This study protocol was reviewed and approved by the Institutional Ethics Committee at Korea University Ansan Hospital (IRB No. 2020AS0041).

Overnight physician-attended standard PSG was carried out using an Alice4 (Respironics, Murrysville, PA, USA) device using the standard neurophysiologic and respiratory signals recommended by the American Academy of Sleep Medicine (AASM). PSG data were manually scored by well-trained sleep technicians and additionally reviewed by certified sleep physicians according to the recommended AASM criteria [2]. We defined OSA as an AHI of $\geq 5$ events/hr.

All study participants underwent CPC analysis using the RemLogic 2.0 CPC analyzer (Embla Systems Inc., San Carlos, CA, USA) during in-laboratory full-night PSG. The CPC data were generated by QRS complex amplitude variations from a single-lead ECG channel, and various parameters were obtained and calculated automatically according to the frequency $[5,7,8]$. The sAHI value was automatically calculated from the analysis of CPC parameters, and non-hypoxic events were calculated from the frequency of coupling during subtypes of elevated low frequency coupling (e-LFC; narrowband-e-LFC [e-LFC $\mathrm{NB}_{\mathrm{NB}}$ or broadband-e-LFC [e-LFC $\left.\left.\mathrm{BB}_{\mathrm{B}}\right]\right)$. The total number of events was divided by the total sleep time and represented as the sAHI [6]. Statistical analysis was carried out using IBM SPSS ver. 21 (IBM Corp., Armonk, NY, USA) and GraphPad Prism 7.0 (GraphPad Software, San Diego, CA, USA).

The Spearman correlation coefficient showed that the sAHI was significantly positively correlated with the AHI $(r=0.973$,

Copyright (C) 2021 by Korean Society of Otorhinolaryngology-Head and Neck Surgery.

This is an open-access article distributed under the terms of the Creative Commons Attribution Non-Commercial License (https://creativecommons.org/licenses/by-nc/4.0) which permits unrestricted non-commercial use, distribution, and reproduction in any medium, provided the original work is properly cited. 
$P<0.001$ ) (Fig. 1). In addition, Bland-Altman analysis found that the AHI and sAHI values showed good agreement and correlations (Supplementary Fig. 1). The mean difference was -2.00 events/hr and the upper and lower limits of agreement were 10.64 and -14.64 , with only $3.09 \%(6 / 194)$ of cases outside of this limit.

The diagnostic value of the sAHI was compared to the standard parameters used to calculate the AHI, and the results are presented in Table 1. The sensitivity and specificity according to OSA severity were $62.16 \%$ and $98.73 \%$ for the controls, $70.83 \%$ and $89.04 \%$ for mild OSA, $51.52 \%$ and $85.71 \%$ for moderate OSA, and $93.67 \%$ and $89.57 \%$ for severe OSA, respectively. Receiver operating characteristic analysis for the diagnosis of OSA based on the sAHI cutoff point of 5.0 events/hr revealed an area under the curve of 0.987 (Supplementary Fig. 2). The diagnostic value was highest for patients with severe OSA. The

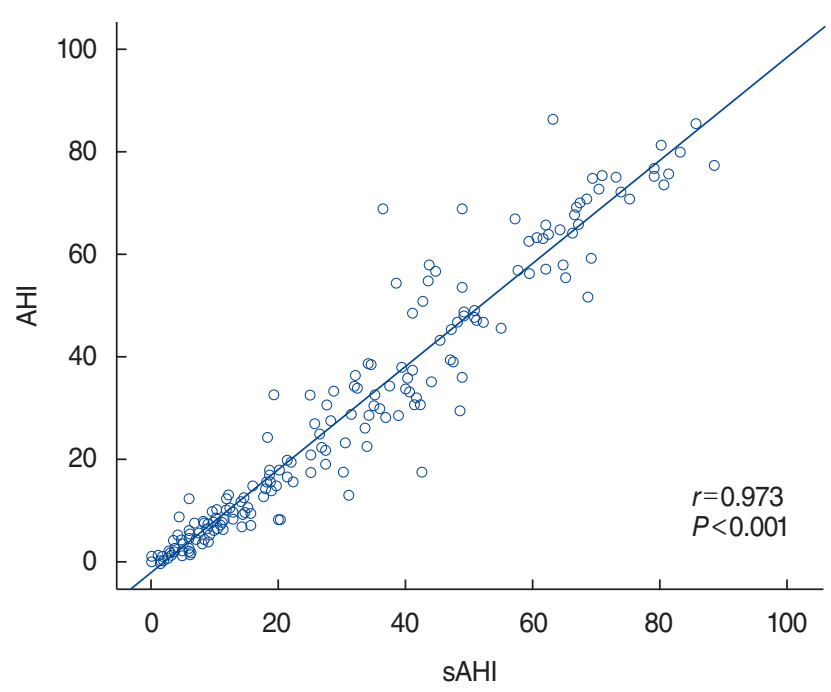

Fig. 1. Correlation coefficients between apnea-hypopnea index (AHI) and software-generated $\mathrm{AHI}$ (sAHI) values. The Spearman correlation method was used to evaluate the relationship between both the parameters, and the degree of correlation (Spearman's rho; $r$ ) was calculated. optimal cutoff values for the diagnosis of OSA using the sAHI were $9.1 / \mathrm{hr}$ (sensitivity, $100 \%$; specificity, $93.63 \%$; $95 \%$ confidence interval, $88.6 \%-96.9 \%$ ).

In this study, we found that the CPC-based sAHI was significantly correlated with the manually-scored AHI. Furthermore, sAHI values can be used to diagnose OSA and to assess its severity with some adjustments. As mentioned above, we previously reported that CPC values closely reflected sleep quality and stability [3,4]. Recently, a cloud-based software technology, SleepImage, has been approved by the Food and Drug Administration [6]. Thus, we could generate sAHI values and assess the diagnostic value of the sAHI in our adult population. Hilmisson et al. [6] recently published an article on the diagnostic value of the sAHI in the pediatric population, and reported strong agreement between the sAHI and AHI in children. In this study, we also found similar results in adult patients with OSA. However, we found that the diagnosis and assessment of OSA using the same AHI values as generated from PSG yielded relatively low sensitivity, except for patients with severe OSA. Therefore, it was necessary to suggest a new sAHI cutoff value for OSA diagnosis, and we found that a sAHI value of 9.1/hr was optimal, yielding a diagnostic accuracy of $100 \%$ sensitivity and $93.63 \%$ specificity.

This study compared respiratory parameters calculated using PSG and CPC. The CPC analysis could be performed using either ECG or photoplethysmography with a portable device [6]; therefore, we consider that this modality might be more convenient than the conventional method. With this convenient diagnostic modality, we can perform multiple tests per day in a patient-friendly sleep environment, such as the patient's own home. Therefore, in light of these advantages, we believe that CPC can be actively used for the diagnosis of sleep-disordered breathing.

In our study, the control and moderate OSA groups contained somewhat fewer patients than the severe OSA group, which may have affected the findings regarding diagnostic accuracy in our study groups.

In conclusion, we suggest that this simple diagnostic modality using CPC might be an alternative to standard PSG for

Table 1. Diagnostic value of CPC-based sAHI compared to AHI

\begin{tabular}{|c|c|c|c|c|c|c|c|c|}
\hline \multirow[t]{2}{*}{ Variable } & \multicolumn{2}{|c|}{$\begin{array}{c}\text { Control }(n=37), \\
A H I<5\end{array}$} & \multicolumn{2}{|c|}{$\begin{array}{l}\text { Mild OSA }(n=49) \\
5 \leq A H I<15\end{array}$} & \multicolumn{2}{|c|}{$\begin{array}{c}\text { Moderate OSA }(n=29) \\
15 \leq A H I<30\end{array}$} & \multicolumn{2}{|c|}{$\begin{array}{c}\text { Severe OSA }(n=79) \\
\quad A H I \geq 30\end{array}$} \\
\hline & Negative & Positive & Negative & Positive & Negative & Positive & Negative & Positive \\
\hline \multicolumn{9}{|l|}{ sAHI } \\
\hline Negative & 155 & 14 & 130 & 14 & 138 & 16 & 103 & 5 \\
\hline Positive & 2 & 23 & 16 & 34 & 23 & 17 & 12 & 74 \\
\hline Sensitivity (\%) & 62.16 & & 70.83 & & 51.52 & & 93.67 & \\
\hline Specificity (\%) & 98.73 & & 89.04 & & 85.71 & & 89.57 & \\
\hline Accuracy (\%) & 91.75 & & 84.54 & & 79.90 & & 91.24 & \\
\hline PPV (\%) & 92.00 & & 68.00 & & 42.50 & & 86.05 & \\
\hline NPV (\%) & 91.72 & & 90.28 & & 89.61 & & 95.37 & \\
\hline
\end{tabular}

CPC, cardiopulmonary coupling; sAHI, software-derived apnea-hypopnea index; OSA, obstructive sleep apnea; PPV, positive predictive value; NPV, negative predictive value. 
OSA diagnosis in adult patients for whom standard PSG is not feasible.

\section{CONFLICT OF INTEREST}

No potential conflict of interest relevant to this article was reported.

\section{ACKNOWLEDGMENTS}

Mr. Hugi Hilmisson, the Director of Research \& Development at

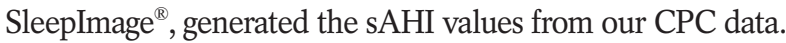

This study was supported by National Research Foundation of Korea (NRF) grant funded by Korean government (Ministry of Education) (NRF-2020R1I1A1A01063604 to Min Young Seo).

\section{ORCID}

Min Young Seo https://orcid.org/0000-0002-1287-6961

Jun Yoo https://orcid.org/0000-0002-9419-7785

Sun Jin Hwang https://orcid.org/0000-0001-9221-0790

Seung Hoon Lee https://orcid.org/0000-0001-7811-2692

\section{AUTHOR CONTRIBUTIONS}

Conceptualization: SHL. Data curation: MYS, JY, SJH. Funding acquisition: MYS. Methodology: SHL. Writing-original draft: MYS.Writing-review \& editing: SHL, MYS.

\section{SUPPLEMENTARY MATERIALS}

Supplementary materials can be found via https://doi.org/10. 21053/ceo.2020.01984.

\section{REFERENCES}

1. Seo MY, Lee JY, Hahn JY, Ryu G, Hong SD, Dhong HJ, et al. Association of obstructive sleep apnea with subclinical cardiovascular disease predicted by coronary artery calcium score in asymptomatic subjects. Am J Cardiol. 2017 Aug;120(4):577-81.

2. Iber C, Ancoli-Israel S, Chesson AL Jr, Quan SF. The AASM manual for the scoring of sleep and associated events. Westchester, IL: American Academy of Sleep Medicine; 2007.

3. Seo MY, Han MS, Jeong YJ, Lee MK, Park S, Hwang SJ, et al. Variation in sleep stability with differences in severity of sleep-disordered breathing in children. Laryngoscope. 2020 May 30 [Epub]. https:// doi.org/10.1002/lary.28769.

4. Seo MY, Hwang SJ, Nam KJ, Lee SH. Significance of sleep stability using cardiopulmonary coupling in sleep disordered breathing. Laryngoscope. 2020 Aug;130(8):2069-75.

5. Thomas RJ, Mietus JE, Peng CK, Goldberger AL. An electrocardiogram-based technique to assess cardiopulmonary coupling during sleep. Sleep. 2005 Sep;28(9):1151-61.

6. Hilmisson H, Berman S, Magnusdottir S. Sleep apnea diagnosis in children using software-generated apnea-hypopnea index (AHI) derived from data recorded with a single photoplethysmogram sensor (PPG): results from the Childhood Adenotonsillectomy Study (CHAT) based on cardiopulmonary coupling analysis. Sleep Breath. 2020 Dec; 24(4):1739-49.

7. Thomas RJ, Mietus JE, Peng CK, Gilmartin G, Daly RW, Goldberger AL, et al. Differentiating obstructive from central and complex sleep apnea using an automated electrocardiogram-based method. Sleep. 2007 Dec;30(12):1756-69.

8. Thomas RJ, Mietus JE, Peng CK, Guo D, Gozal D, MontgomeryDowns H, et al. Relationship between delta power and the electrocardiogram-derived cardiopulmonary spectrogram: possible implications for assessing the effectiveness of sleep. Sleep Med. 2014 Jan; 15(1):125-31. 